\title{
Non-Parametric Operational Modal Analysis Methods in Frequency Domain: A Systematic Review
}

\author{
Elsa María Cárdenas ${ }^{1, *}$, Luis Ulises Medina ${ }^{2}$ \\ ${ }^{1}$ Departamento de Tecnología Industrial, Universidad Simón Bolívar, Venezuela \\ ${ }^{2}$ Instituto de Diseño y Métodos Industriales, Facultad de Ciencias de la Ingeniería, Universidad Austral de Chile, Chile \\ Received 28 July 2020; received in revised form 30 September 2020; accepted 02 November 2020
}

DOI: https://doi.org/10.46604/ijeti.2021.6126

\begin{abstract}
The objective of this research is to present a systematic review of the non-parametric modal analysis methods in the frequency domain. Peak picking (PP), frequency domain decomposition (FDD), enhanced frequency domain decomposition (EFDD), and frequency-spatial domain decomposition (FSDD) are revisited and didactically illustrated by means of modal identification for a study case proposed in previous researches. Algorithm schemes are illustrated to summarize these frequency domain OMA techniques. Modal frequencies, modal damping ratios, and modal shapes are estimated using the different OMA techniques and compared to estimations obtained by the free decay (FD) method reported in previous researches. These are employed to compare the results obtained by the methods presented herein and show a very good correlation in obtaining modal frequencies and a low correlation in the case of modal damping.
\end{abstract}

Keywords: operational modal analysis, peak picking, frequency domain decomposition, enhanced frequency domain decomposition, frequency-spatial domain decomposition

\section{Introduction}

Operational modal analysis (OMA) arises as a response to the need to identify the modal parameters of the structures, which may be difficult, expensive, or restricted for conventional experimental modal analysis (EMA). OMA is addressed in the modal parameters identification for systems subjected to one or more excitation sources, which cannot be measured [1-2]. These excitation signals are produced in normal operating conditions of the system under environmental forces [3]. Thus, the system response is the only information available for identification purposes [4-5]. OMA has been widely used in civil engineering since the $1990 \mathrm{~s}$ as environmental tests [3, 6-7]. Due to the advantages provided by these methods, their use has spread to other areas such as aerospace engineering [8-10] and mechanical engineering [11-15], as well as monitoring of equipment for preventive maintenance [6, 16-17].

OMA methods obtain information physically related to structure from correlation functions and spectral densities [2, 4]. The methods available to perform these analyses can be carried out in the time domain and the frequency domain. In the time domain identification in OMA, the information is extracted from the correlation functions [18]. The biggest drawback of using these identification techniques is that all modes contribute to the range of the problem at any time when working with free decays, usually estimated as correlation functions. However, it is possible to obtain bias-free data [4].

Frequency domain methods start with estimating the output response spectrum. The main advantage of these methods is the simplicity and speed with which the modal parameters can be obtained at a very low computational cost, compared to those

* Corresponding author. E-mail address: elsacardenas@usb.ve

Tel.: +58-416-8044603 
in the time domain. [3]. Employing a frequency domain approach allows a natural division of the information in the data to identify the modes of interest, and simplifying the identification model since it would only be necessary to model the modes in the selected frequency band [19-20]. However, the results of this scheme depend directly on the resolution of the estimation of the power spectral density (PSD), which suffers some type of bias [4, 20-21].

These methods can be parametric, when it is assumed that the sampled data comes from a specific probability distribution or; in contrast, these methods can be non-parametric, when it is assumed that the distribution from which the sample comes cannot be defined a priori [2].

The parametric approaches can be used only when there is enough information about the studied signal, which allows the formulation of a model. These methods may offer more accurate spectral estimations (inputs to the OMA methods) than the non-parametric ones in cases where the data effectively satisfies the model assumed by the previous methods. However, "in the more likely case that the data do not satisfy the assumed models and the non-parametric methods may outperform the parametric ones owing to the sensitivity of the latter to model misspecifications" [22].

The literature presents reviews articles of different OMA methods [2, 3, 6], and some of them show cases of the interest that allow comparing the results obtained between the different methods [7, 20]. In this research, it is intended to condense only non-parametric OMA methods in the frequency domain, which explains their use in a simple way. The objective of this research is to present a systematic review of the non-parametric modal analysis methods in the frequency domain, which shows the advantages and disadvantages of their use when obtaining the modal parameters of a system. This paper shows under what conditions these methods can be used and what results can be obtained when applied in the identification of a system.

A classical two-degree-of-freedom mass-spring-damper system is tackled as a case study. The modal parameters of this system; modal frequencies, modal damping ratios, and modal shapes are estimated, by means of different frequency domain techniques and the results are compared with those ones obtained by the free decay (FD) technique [23].

\section{Operational Modal Analysis}

The development of these methods, in the time domain and the frequency domain, has evolved since the 90s. Towards 1992, the natural excitation technique (NExT) was developed by James \& Carne $[11,24]$. They proposed that the correlation function is the addition of decaying sinusoids, each one with a damped natural frequency and a damping ratio related to a modal form of the structure [11, 24]. As an extension of the EMA in 1993, Felber [25] applied this technique in the evaluation of the dynamic characteristics of a bridge and compared its results with those obtained using modal analysis techniques, concluding that the modal parameters could be obtained even with very low excitations. Then, James, Carne, and Lauffer [26] published a new theoretical derivation of NExT, which compares the experimental results calculated using NExT with analytical predictions of damping using aeroelastic theory. This study concludes that NExT provides the necessary information to refine analytical predictions. However, a difference in the expected damping in close modes is observed, due to the inability of NExT to separate symmetric and anti-symmetric modes [26].

The basic frequency domain method is the peak picking (PP) technique, based on the relationship between the input and output of PSD in a random process [3, 27-28]. In this method, the natural frequencies are obtained directly from the peaks present in the PSD function. This basic technique provides reliable results for well-separated modes, though it is limited bythe resolution of the PSD, the extraction of the operational form of deviation (ODS) instead of the modal form of the system, the low precision in damping ratio's calculation, and the restriction when dealing with systems with closing modes $[3,6]$.

In 2000, Brincker, Zhang, and Andersen [29] published an extension of the PP method. Those authors proposed a singular value decomposition of the PSD matrix into a set of functions of auto-spectral density each corresponding to a degree-of-freedom (DOF). They presented the development of this technique, which they named frequency domain 
decomposition (FDD). They simulated the response of a two-storey building loaded with noise and closing modes. This response was analyzed using the FDD technique, the natural frequencies, damping ratios, and mode shapes were identified with high accuracy [29]. Later, in 2001, Bricker, Ventura, and Andersen presented the enhanced frequency domain decomposition (EFDD) method. In this method, the singular values (SV) in the vicinity of the natural frequencies are transferred back to the time domain using the inverse of the fast Fourier transform (IFFT) and the damping coefficients are obtained by using logarithmic decrease techniques [30-31].

To obtain more precise results, Zhang [33] proposed the frequency-spatial domain decomposition (FSDD) method in 2005 [32]. This method used the property of the unitary singular matrix to derive an enhanced output PSD, using as a modal filter the singular vector, corresponding to the $k$-th damped natural frequency. Using this technique, modal frequencies and damping ratios are estimated from the enhanced PSD directly, without the need to perform IFFT [34].

\subsection{The peak picking $(P P)$ method}

The PP method is the simplest and fastest approximation technique known for estimating the modal properties of a vibrating structure, measuring only the response of the system [2]. This technique provides good estimates for lightly damped systems with well separated modes [6].

In the technique, it is assumed that the unknown input excitation can be characterized as a zero-mean Gaussian white noise, as all the methods under the frequency domain start from obtaining the spectral density function of response, previously estimated from the system responses [2]. If $y_{\mathrm{n}}$ is a measured system response at time $t_{\mathrm{n}}\left\{\left(y_{1}, t_{1}\right),\left(y_{2}, t_{2}\right) \ldots . .\left(y_{\mathrm{n}}, t_{\mathrm{n}}\right)\right\}$, the unknown excitation that has generated this response $x_{\mathrm{n}}$; thus, it is considered like Gaussian white noise [31]. Then, the estimation of the spectrum matrix from the output measurements can be obtained by using the Welch method [25]. If the system response has been measured at $m$ locations on the system, $y_{\mathrm{n}}$ is an $m \times 1$ vector. Assuming that Gaussian white noise excitation has been applied in $r$ location, then, $x_{\mathrm{n}}$ is a $r \times 1$ vector, the $m \times m$ spectral density function $S_{y y}(\quad)$ can be defined as in Eq. (1) [31].

$$
S_{Y Y}(\omega)=H^{*}(\omega) R_{u u} H^{T}(\omega)
$$

where $H^{*}(\omega)$ and $H^{T}(\omega)$ are the complex conjugates of the frequency response function and their transposition respectively, while $R_{u u}$ denotes a scalar constant input matrix, which is assumed to be white noise.

The PP method is based on the fact that the frequency response function (FRF) of a given system will experience extreme values around the modal frequencies of the system [35]. In this way, the natural frequency response is dominated by the corresponding modal form and the peak in the frequency spectrum can be used to identify modal frequencies [29], which correspond to peaks on the averaged and normalized spectral density function.

This method is the most suitable for making a first check on the quality of the data collected and obtaining a first approach to the dynamic properties of the system since it is a non-parametric method in the frequency domain [2].

\subsection{Frequency domain decomposition (FDD)}

An alternative method known as FDD was proposed by Brincker, Zhang, and Andersen [29], which allows overcoming some of the issues related to the modal identification by the PP method. This method is an extension of the classical approach technique in the frequency domain.

The method maintains most of the advantages of PP, such as peak selection. However, the FDD technique approximates the decomposition of the spectral density matrix into a set of systems of auto-spectral functions, each of which corresponds to an one-DOF system utilizing the singular value decomposition (SVD) technique [36-37]. This allows the closing modes to be separated so that they can be estimated with a very good approximation. 
Taking into account that the structure is lightly damped, then the $R_{u и}$ input is uncorrelated and therefore a diagonal matrix. Furthermore, the modal shapes are orthogonal, Eq. (1) can be simplified as Eq. (2) [2, 29],

$$
S_{Y Y}(\omega)=\mathrm{VC}(\omega) \mathrm{V}^{T}
$$

where $C(\omega)$ is a diagonal matrix composed of functions of $\omega$, each one dependent on the natural frequency and the modal damping ratio of the structure, and V is a matrix whose columns represent the modal forms. The next step is to extract the singular values and vectors of the spectral density of the response using the decomposed singular values of the matrix $\hat{S}_{y y}(\omega)$, as shown in Eq. (3),

$$
\hat{S}_{Y Y}(\omega)=U_{j} S_{j} U_{j}^{H}
$$

where the matrix $U_{j}=u_{j 1}, u_{j 2}, \ldots, u_{j \mathrm{n}}$ is the unit matrix containing the singular vectors $u_{j}, U_{j}^{H}$ the Hermitian matrix of $U_{j}$, the matrix $U_{j}$ is an orthonormal matrix $U_{j} U_{j}^{H}=I$, and $S_{j}$ is a diagonal matrix that contains the scalar singular values [4].

To identify the modes that contribute to the system response at a certain frequency, the proximity to the peak corresponding to the $k$-th mode in the spectrum should be used. This mode or a possible near mode will be dominant. Therefore, according to the FDD theory, the first singular vector is an estimator of the modal form $\widehat{\Phi}=u_{j 1}[4]$.

The SVD provides the singular values in an ascending way, which means that for each discrete value of $\omega$, the first singular value contains an ordinate of the spectrum associated with the dominant mode at that frequency, and the corresponding singular value is the auto spectral density function of the corresponding system's DOF [4]. The number of non-zero singular values represents the range of the spectrum matrix at a specific frequency. In other words, the number of modes with a significant contribution to the system response at that particular frequency [2].

The singular values of the spectrum associated with each mode can be selected by comparing the singular vectors related to points close to an identified resonance frequency, with the singular vector related to the peak at this frequency (the estimated modal shape $\widehat{\Phi}$ ). This selection is generally made by setting a limit for a "modal assurance criterion" (MAC), which measures the correlation between two modal forms $\left(\widehat{\Phi}_{1}, \widehat{\Phi}_{2}\right)$. This correlation is given by Eq. (4) [2].

$$
\operatorname{MAC}_{\hat{\Phi}_{1} \hat{\Phi}_{2}}=\frac{\left(\hat{\Phi}_{1}^{T} \Phi_{2}\right)^{2}}{\left(\hat{\Phi}_{1}^{T} \Phi_{1}\right)\left(\hat{\Phi}_{2}^{T} \Phi_{2}\right)}
$$

The index of the MAC function varies between 0 and 1 when the modes only differ by a scale factor and the modes are orthogonal, respectively. The modal frequencies can be clearly visible as peaks by the singular values of the decomposed spectral density function [33].

\subsection{Enhanced frequency domain decomposition (EFDD)}

The second generation of FDD, called EFDD, has been developed to estimate frequencies and modal shapes; also, it has been added estimation of damping ratios. In this technique, the data of singular values close to the peak with the corresponding singular vector are transformed to the time domain using the IFFT [4], which provides an approximation to the correlation function of the one DOF system.

The singular values near the peak, with the corresponding singular vector with a high MAC value (above a user-specified MAC rejection level) [38], are transferred back to the time domain by IFFT. Using the FD, the time domain function (which is also the auto correlation function of the single degree-of-freedom (SDOF) system of the $k$ - $t h$ mode), the natural frequency, and 
the damping ratio can be identified [31]. The modal damping ratio, $\zeta$, can be found by the logarithmic decrement technique from the logarithmic envelope of the correlation function [31, 39], defined as shown in Eq. (5) [40], where $x_{1}$ and $x_{2}$ are two consecutive amplitudes obtained from the time domain response.

$$
\delta=\ln \frac{x_{1}}{x_{2}}=\frac{2 \pi \zeta}{\sqrt{1-\zeta^{2}}}
$$

One way to obtain a result with a lower degree of error is to apply a linear regression between the logarithm of the values of peaks and valleys obtained from the time domain response and the logarithmic decrease, as proposed in Eq. (6), where $z_{j}$ is the logarithm of the amplitudes $\ln \left(x_{j}\right), a$ is the slope of the curve that will correspond to the negative of the logarithmic decrease $-\delta, y_{j}$ is the term $j-1$, and $b$ is the cut of the line with the $x$ axis corresponding to the logarithm of the first amplitude ln $\left(x_{j}\right)$ [41].

$$
z_{j}=a_{j} y_{j}+b
$$

Knowing the logarithmic decrement, therefore, Eq. (7) can be employed to determine the damping ratio.

$$
\zeta=\frac{\delta}{\sqrt{\delta^{2}+4 \pi^{2}}}
$$

Eq. (8) is employed to obtain the natural frequency $f$ [42], where $f_{d}$ is the damped natural frequency.

$$
f=\frac{f_{d}}{\sqrt{1+\zeta^{2}}}
$$

This can be attained by a linear regression between the points called "zero crossing", which correspond to the interception of the auto-correlation function with the time axis (consequently, the value of the auto-correlation function at these points are zero), and the time corresponding to the extremes (peaks) of function [31, 33, 37, 42].

The algorithm for the use of this technique can be summarized as follows [33]:

(1) Identify the modal PSD function close to the resonance peaks.

(2) Using the IFFT, transform each function from one DOF to the time domain.

(3) Obtain the natural frequencies; determine the "zeros crossing" as a function of time.

(4) Obtain the damping estimation by the logarithmic decrement technique and the logarithmic envelope of the correlation function.

In this damping estimation, only the data near the peak of the singular value plot are utilized for the IFTT to calculate approximate correlation functions of the corresponding SDOF system, which may cause bias errors in the damping estimation. Furthermore, when dealing with closely spaced modes, the beating phenomenon can occur, which can lead to a damping ratio imprecise estimation when the logarithmic decrement technique is employed [33].

\subsection{Frequency-spatial domain decomposition (FSDD)}

The FSDD method consists of the third generation of FDD and it has been proposed to further improve FDD performance. The FSDD method uses the property of a unitary singular matrix to derive enhanced output spectral density matrix $e \hat{S}_{y y}(\omega)$ by modal filtering. Eq. (9) defines an enhanced spectrum for the $k$-th mode [33-34]. 


$$
e \hat{S}_{Y Y}(j \omega)=u_{1}^{H}\left(\omega_{m}\right) \times S_{Y Y}(j \omega) \times u_{1}\left(\omega_{m}\right) \approx \operatorname{Re}\left(\frac{2 C \cdot m}{i \omega-\lambda}\right)
$$

In this way, the output PSD is enhanced in the vicinity of the $k$-th modal frequency and this behaves like one DOF system. The singular vector corresponding to a modal frequency acts as a modal filter.

The least square algorithm can be employed to obtain the real and imaginary parts of the pole $\lambda_{m=}-\sigma_{m} \pm i \omega_{d m}$, utilizing the enhanced output PSD data of the spectrum lines in the vicinity of modal frequency and by applying the curve fitting to the enhanced spectrum plot. The damping factor and the damped modal frequency are $\sigma_{m}$ and $\omega_{d m}$, respectively. The modal frequency and the damping ratio can be obtained using Eqs. (10) and (11) [33].

$$
\begin{aligned}
& \omega_{m}=\sqrt{\sigma_{m}^{2}+\omega_{d m}{ }^{2}} \\
& \zeta=\sigma_{m} / \sqrt{\sigma_{m}^{2}+\omega_{d m}{ }^{2}}
\end{aligned}
$$

Fig. 1 summarizes the algorithm schemes for the frequency domain-based techniques mentioned in this compilation that has been integrated.

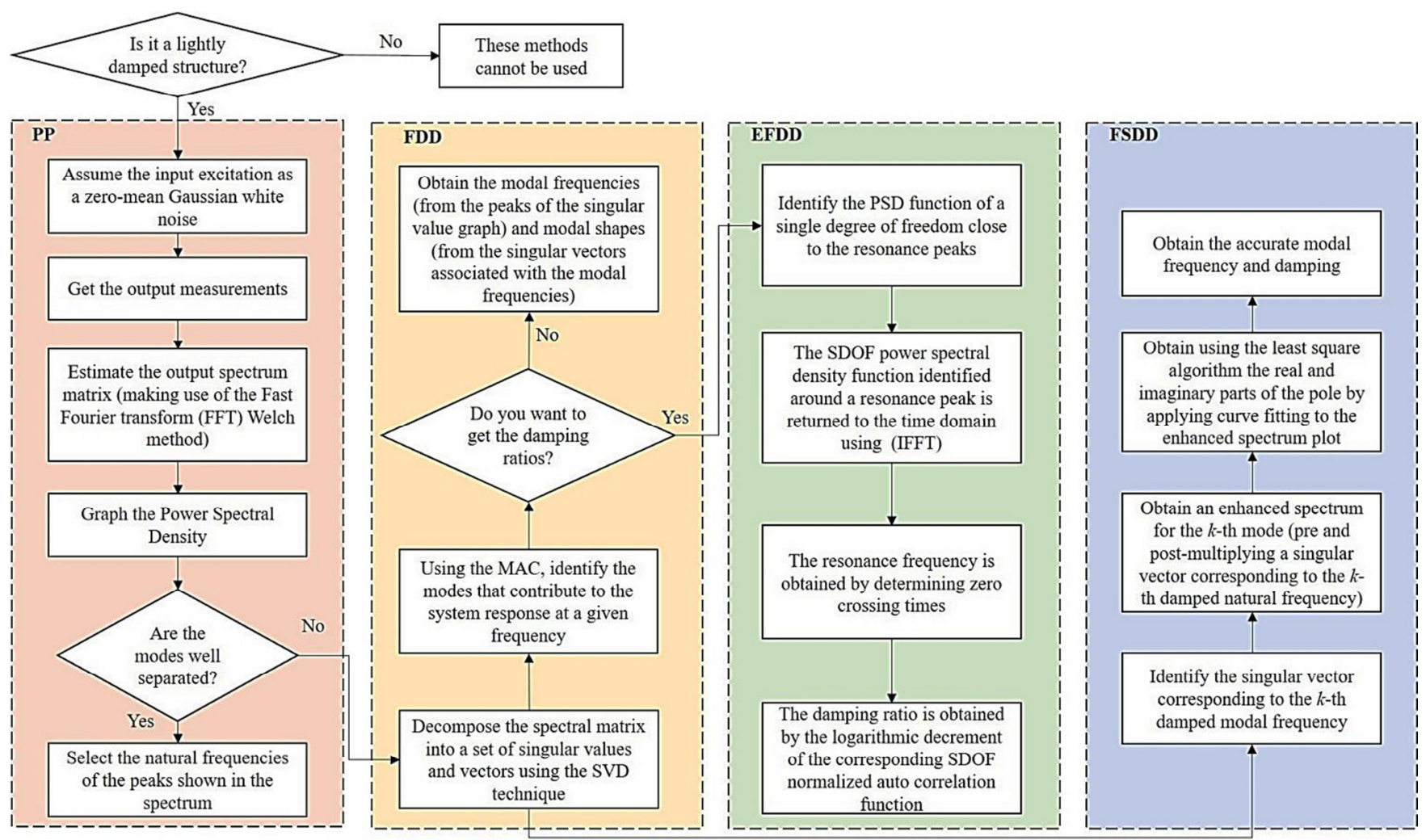

Fig. 1 Summary algorithm of OMA techniques in frequency domain, PP, FDD, EFDD and FSDD

\section{Case Study}

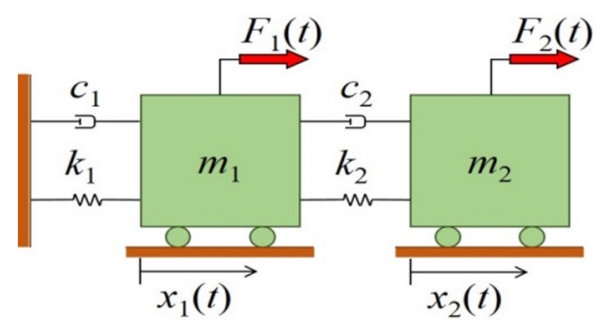

Fig. 2 Shock absorber spring mass system 
A case study is proposed to demonstrate the use of these methods in a simple example that is presented in the literature [23]. This consists of a two-degree-of-freedom system shown in Fig. 2. Asmussen considered two degrees of freedom for this case because only the structural modes with an eigenfrequency within that frequency range where the forces workare into vibration; therefore, the frequencies associated with this system are finite and can be identified [23].

The system has the following characteristics; mass matrix; [ $10 ; 01] \mathrm{kg}$, stiffness matrix $K=\left[k_{11} ; k_{12}, k_{21} ; k_{22}\right]=[700-200$; -200 500] N/m and damping matrix $C=\left[c_{11} ; c_{12}, c_{21} ; c_{22}\right]=1.5 \times[0.9-1 ;-11.8] \mathrm{N} \cdot \mathrm{s} / \mathrm{m}$. In here, the system is loaded by uncorrelated Gaussian white noise processes at each mass, the sampling rate chosen is $120 \mathrm{~Hz}$, and the response is simulated using ARMAV models [23].

Table 1 shows the modal parameters obtained using the FD technique by Asmussen [23]. These parameters are used as a reference in the comparison of the results obtained using the study techniques presented.

Table 1 Modal system parameters obtained using the FD technique by Asmussen [23]

\begin{tabular}{|c|c|c|c|c|}
\hline & Frequency $(\mathrm{Hz})$ & $\zeta(\%)$ & $|\Phi|^{1}$ & $|\Phi|^{2}$ \\
\hline Mode 1 & 3.09 & 1.69 & 1 & 1.61 \\
\hline Mode 2 & 4.56 & 3.56 & 1 & 0.62 \\
\hline
\end{tabular}

While the system previously described was excited in [23] with Gaussian mean white noise, for the proposed case study, it has been excited by adding an initial displacement for mass 2 of $1 \mathrm{~m}$ to the right. Fig. 3 illustrates the response to excitation of the system shown in Fig. 2, which has been obtained utilizing a $4^{\text {th }}$ order Runge-Kutta method. These results are used as input to the operational modal analysis methods presented.


Fig. 3 System response to excitation

The characteristics of the chosen system meet the hypotheses necessary for the proper development of the presented methods, well separated modal frequencies, and low damping, as a result, good estimates of the modal parameters obtained from these responses are expected.

\section{Results and Discussion}

Based on the response shown in Fig. 3, assuming that the input excitation is unknown, and also characterizing it as a zero-mean Gaussian white noise, the PSD is obtained by the Welch method using a rectangular window and overlapping of 50 $\%$ and a sample rate of $4000 \mathrm{~Hz}$. Fig. 4 shows the PSD of the response system. Two dominant modes in the frequency range of 0 to $6 \mathrm{~Hz}$ are identified with the PP algorithm. The peaks in the spectrum corresponding to the modal frequencies of the system are observed at the frequencies: $3.08 \mathrm{~Hz}(19.35 \mathrm{rad} / \mathrm{s})$ and $4.578 \mathrm{~Hz}(28.764 \mathrm{rad} / \mathrm{s})$ for the first and second mode, respectively.

Using the FDD technique, the estimated spectral density matrix has been approximated to an auto-spectral function by means of the SVD technique. Employing the identified SDOF auto spectral density function associated to the first degree of 
freedom, it was obtained the singulars values around the two peaks with a MAC value equal to 0.95. Fig. 5 illustrates the auto-spectral function and the singular values related to the first and second modes. The resultant modal frequencies are equal to $3.08 \mathrm{~Hz}(19.35 \mathrm{rad} / \mathrm{s})$ and $4.578 \mathrm{~Hz}(28.76 \mathrm{rad} / \mathrm{s})$ for the first and second mode, respectively.

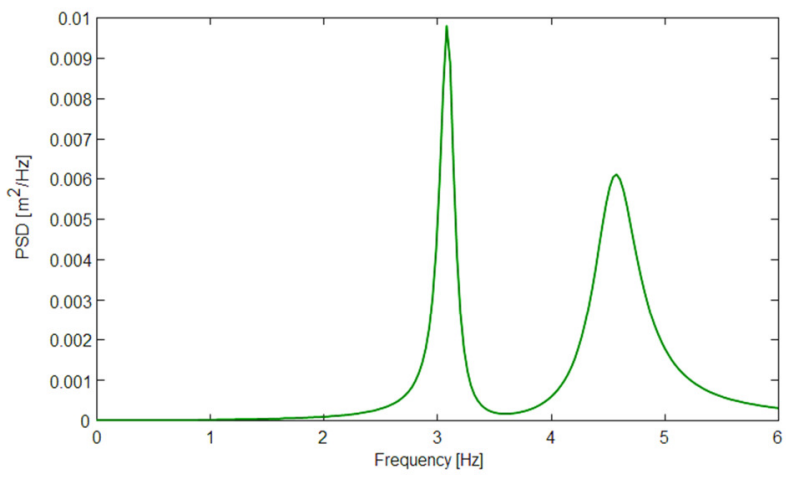

Fig. 4 Spectral Density

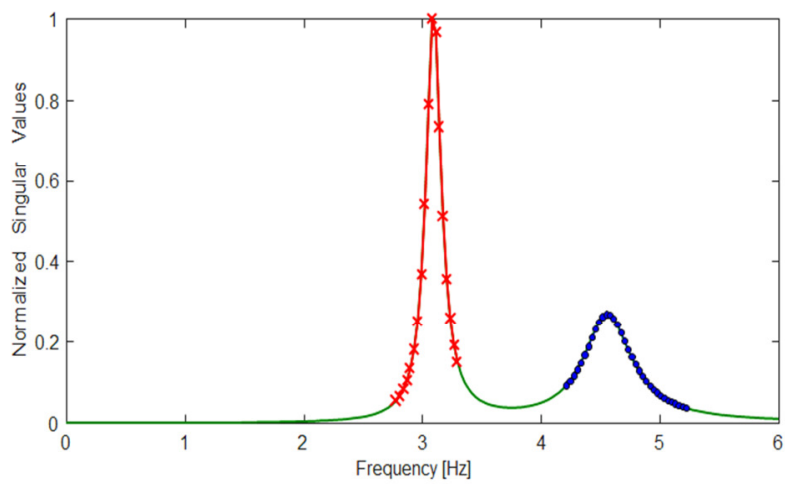

Fig. 5 Values associated with MAC 0.95, for the peaks of the PSD

The EFDD technique was applied to obtain the damping ratios. The singular values close to the peak were transformed back to the time domain by IFFT. The damping ratios were obtained by the logarithmic decrement of these responses (displayed in Fig. 6) for each mode, and these damping ratios are 1.19\% and $2.79 \%$ for the first and second mode, respectively.
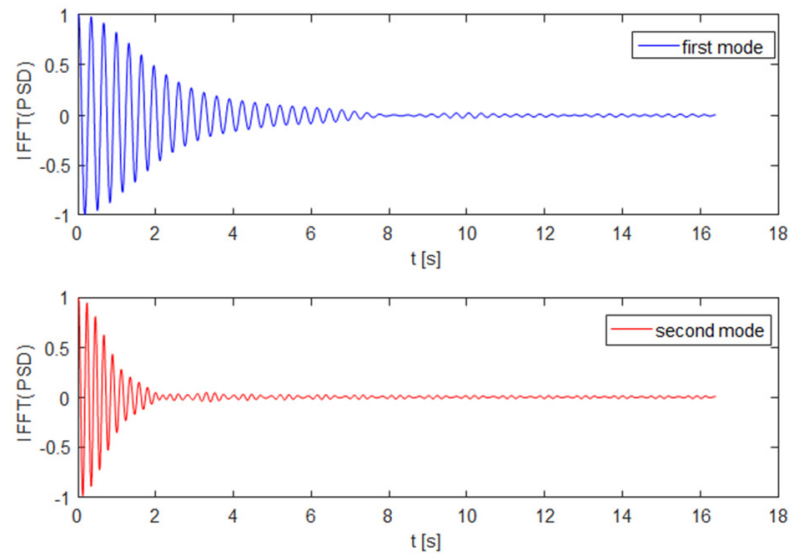

Fig. 6 IFFT of the 2GL system using filtered MAC values 0.95

The spectral lines (one DOF per mode) corresponding to the enhanced output PSD have been obtained using the singular vectors of the identified frequencies. Fig. 7 demonstrates the modal frequencies obtained using the FSDD algorithm. These are $3.05 \mathrm{~Hz}(19.16 \mathrm{rad} / \mathrm{s})$ for the first mode (Fig. 7(a)) and $4.52 \mathrm{~Hz}(28.40 \mathrm{rad} / \mathrm{s})$ for the second mode (Fig. 7(b)). The damping ratios were achieved by the method of least square curve fitting on the enhanced spectrum. They are $1.14 \%$ and $2.77 \%$ for the first and second mode, respectively.

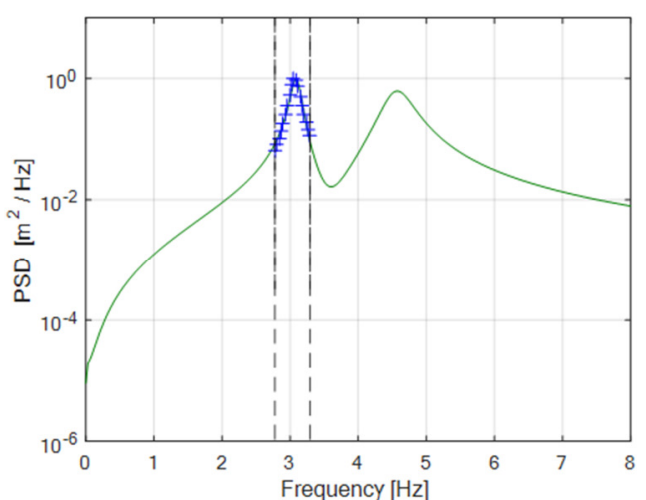

(a) PSD

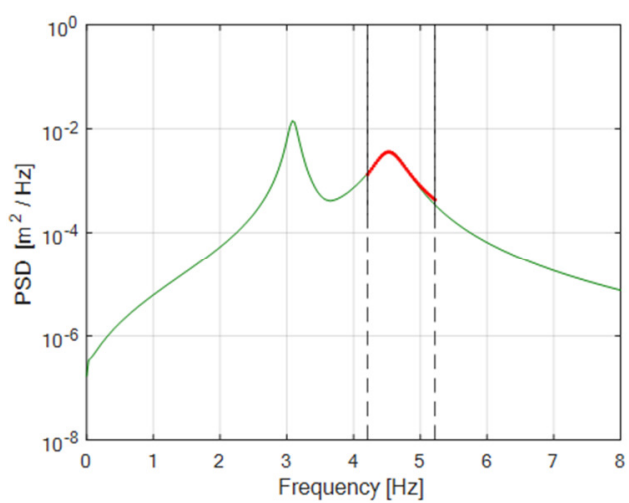

(b) FSDD

Fig. 7 Identification of the enhanced output 
Tables 2 and 3 present the modal parameters obtained using each of these methods. Table 2 indications the undamped natural frequency in $\mathrm{Hz}$ and the damping ratios for each of the techniques used herein, while Table 3 shows the normalized modal forms obtained by each of these techniques.

Table 2 Comparison between the frequency and damping ratio values obtained by the different OMA techniques

\begin{tabular}{|c|c|c|c|c|c|c|c|c|c|c|}
\hline & \multicolumn{4}{|c|}{ Frequency (Hz) } & \multicolumn{4}{c|}{ Damping ratio $\zeta(\%)$} \\
\hline Technique & FD & PP & FDD & EFDD & FSDD & FD & PP & FDD & EFDD & FSDD \\
\hline Mode 1 & 3.09 & 3.08 & 3.09 & 3.10 & 3.05 & $1.69 \%$ & - & - & $1.19 \%$ & $1.14 \%$ \\
\hline Mode 2 & 4.56 & 4.58 & 4.55 & 4.51 & 4.52 & $3.56 \%$ & - & - & $2.79 \%$ & $2.77 \%$ \\
\hline
\end{tabular}

Table 3 Comparison of the mode shapes obtained by the different OMA techniques.

\begin{tabular}{|c|c|c|c|c|}
\hline & \multicolumn{5}{|c|}{ Mode Shape } \\
\hline Technique & FD & FDD & EFDD & FSDD \\
\hline Mode 1 & {$\left[\begin{array}{ll}1 & 1.61\end{array}\right]$} & {$\left[\begin{array}{ll}1 & 1.46\end{array}\right]$} & {$\left[\begin{array}{ll}1 & 1.46\end{array}\right]$} & {$\left[\begin{array}{ll}1 & 1.46\end{array}\right]$} \\
\hline Mode 2 & {$\left[\begin{array}{ll}1 & 0.62\end{array}\right]$} & {$\left[\begin{array}{ll}1 & 0.59\end{array}\right]$} & {$\left[\begin{array}{ll}1 & 0.61\end{array}\right]$} & {$\left[\begin{array}{ll}1 & 0.61\end{array}\right]$} \\
\hline
\end{tabular}

It can be observed in Table 2 that the values of the undamped natural frequency achieved by the different techniques are in good correlation with those obtained by the FD technique presented by Asmussen [23]. The standard deviations for the first mode 1 is equal to \pm 0.0192 and for the second mode is \pm 0.0288 , being the FDD technique the most approximate for this case study. For the damping ratio, there is no good correlation, and the values of standard deviation are \pm 0.259 and \pm 0.388 for the first and second mode, respectively, as expected according [29-35].

The modal forms have been obtained using the FDD, EFDD and FSDD techniques, their results demonstrate a very good correlation concerning the values used as reference.

\section{Conclusions}

A systematic review of the non-parametric methods of modal analysis in the frequency domain, such as PP, FDD, EFDD, and FSDD, was presented in this research, showing the advantages and disadvantages of their use, under what conditions these methods can be used, and the expected results when these are applied in system identification.

A scheme summarized the algorithms based on OMA techniques in the frequency domain mentioned in this compilation. A case study shows the use of these methods to obtain the parameters of a shock absorber mass system of two degrees of freedom with modal parameters identified in previous researches, which were employed to compare the results obtained by the methods presented herein. The results show a very good correlation in obtaining modal frequencies and a low correlation in the case of modal damping.

\section{Acknowledgements}

First author expresses her recognition to Universidad Simón Bolívar. The second author acknowledged the support given by the Instituto de Diseño y Métodos Industriales, Facultad de Ciencias de la Ingeniería (Universidad Austral de Chile).

\section{Conflicts of Interest}

The authors declare no conflict of interest.

\section{References}

[1] J. Debille, S. Pauwels, and B. Peeters, "The Benefits of Operational Modal Analysis of Aircraft and Spacecraft Structures," European Test \& Telemetry Conference (ETTC 2005), June 2005, pp. 1-6.

[2] B. Peeters and G. De Roeck, "Stochastic System Identification for Operational Modal Analysis: A Review," Journal of Dynamic Systems, Measurement, and Control, vol. 123, no. 4, pp. 659-667, December 2001. 
[3] M. H. Masjedian and M. Keshmiri, “A Review on Operational Modal Analysis Researches: Classification of Methods and Applications,” 3rd International Operational Modal Analysis Conference (IOMAC 2009), May 2009, pp. 707-716.

[4] R. Brincker and C. E. Ventura, Introduction to Operational Modal Analysis, Hoboken, New Jersey: John Wiley \& Sons, 2015.

[5] Z. Xia, T. Wang, and L. Zhang, "Detection and Removal of Harmonic Components in Operational Modal Analysis," Journal of Vibroengineering, vol. 19, no. 7, pp. 5278-5289, November 2017.

[6] G. Morteza and S. Ahmad, "Operational Modal Analysis Techniques and their Theoreticals and Practical Aspect: A Comprehensive Review and Introduction," 6th International Operational Modal Analysis Conference (IOMAC 2015), May 2015, pp. 1-16.

[7] G. Zini, M. Betti, G. Bartoli, and S. Chiostrini, "Frequency vs Time Domain Identification of Heritage Structures," Procedia Structural Integrity, vol. 11, pp. 460-469, 2018.

[8] B. Peeters, T. De Troyer, P. Guillaume, and H. Van der Auweraer, "In-Flight Modal Analysis-A Comparison Between Sweep and Turbulence Excitation," International Conference on Noise and Vibration Engineering (ISMA2006), September 2006, pp. 1627-1642.

[9] A. De Vivo, C. Brutti, and J. L. Leofanti, "Vega In-Flight Modal Identification with the Operational Modal Analysis Technique,” Journal of Spacecraft and Rockets, vol. 51, no. 5, pp. 1464-1473, September 2014.

[10] B. Peeters, S. Manzato, and H. Van der Auweraer, "Solutions to Deal with Harmonics and Noise for Helicopter in-Flight Data Dynamic Identification,” 5th International Operational Modal Analysis Conference (IOMAC 2013), May 2013, pp. $1-8$.

[11] G. James and T. Carne, "Damping Measurements on Operating Wind Turbines Using the Natural Excitation Technique (NExT)," 11th ASME Wind Energy Symposium, January 1992, pp. 75-81.

[12] M. A. Anuar, A. A. M. Isa, A. R. Zamri, and R. Brincker, "Critical Experimental Issues of Cracked Aluminum Beam in Operational Modal Analysis,” Journal of Mechanical Engineering, vol. 15, no. SI 2018 Vol 5-6, pp. 211-225, 2018.

[13] E. P. Carden and M. Lindblad, "Operational Modal Analysis of Torsional Modes in Rotating Machinery," Journal of Engineering for Gas Turbines and Power, vol. 137, no. 2, pp. 022501 (7 pages), February 2015.

[14] E. P. Carden, "Investigation of Offshore Diesel Generator Failure Using Operational Modal Analysis," 5th International Operational Modal Analysis Conference (IOMAC 2013), May 2013, pp. 1-10.

[15] M. Karlsson, H. Samuelsson, and M. Karlberg, "Using Operational Modal Analysis to Determine Rotordynamic Modes," 13th International Symposium on Transport Phenomena and Dynamics of Rotating Machinery (ISROMAC-13), April 2010, pp. 527-530.

[16] A. Mironov, P. Doronkin, A. Priklonsky, and I. Kabashkin, "Operational Modal Analysis (OMA) Application for Condition Monitoring of Operating Pipelines," Transport and Telecommunication Journal, vol. 16, no. 4, pp. 305-319, 2015.

[17] H. Shokravi, H. Shokravi, N. Bakhary, S. S. Rahimian Koloor, and M. Petrů, "Health Monitoring of Civil Infrastructures by Subspace System Identification Method: An Overview," Applied Sciences, vol. 10, no. 8, pp. 1-29, 2020.

[18] A. Bajric, R. Brincker, and C. T. Georgakis, "Evaluation of Damping Using Time Domain OMA Techniques," 2014 SEM Fall Conference and International Symposium on Intensive Loading and Its Effects, October 2014, pp. 1-6.

[19] S. K. Au, "Bayesian Operational Modal Analysis," Identification Methods for Structural Health Monitoring, Berlin: Springer, 2016.

[20] E. Reynders, "System Identification Methods for (Operational) Modal Analysis: Review and Comparison," Archives of Computational Methods in Engineering, vol. 19, no. 1, pp. 51-124, March 2012.

[21] H. Cai, P. Du, H. Liu, J. Guo, and Y. Gao, "Modal Analysis of Drive Axle Based on OMA Time Domain Method Identification," International Conference on Electric Information and Control Engineering (ICEICE 2011), April 2011, pp. 2323-2328.

[22] P. Stoica and R. Moses, Spectral Analysis of Signals, Upper Saddle River, New Jersey: Prentice Hall, 2005.

[23] J. Asmussen, "Modal Analysis Based on the Random Decrement Technique," Ph.D. dissertation, Department of Mechanical Engineering, Aalborg University, Aalborg, Denmark, 1997.

[24] G. H. James, T. G. Carne, J. P. Lauffer, and A. R. Nord, "Modal Testing Using Natural Excitation," 10th International Modal Analysis Conference, February 1992, pp. 1209-1216.

[25] A. Felber, "Development of a Hybrid Bridge Evaluation System," Ph.D. dissertation, Department of Civil Engineering, The University of British Columbia, Vancouver, Canada, 1994.

[26] G. James, T. Carne, and P. Lauffer, "The Natural Excitation Technique (NExT) for Modal Parameter Extraction from Operating Wind Turbines,” Sandia National Laboratories, Technical Report SAND-92-1666, February 01, 1993. 
[27] J. S. Bendat, "Spectral Techniques for Nonlinear System Analysis and Identification," Shock and Vibration, vol. 1, no. 1, pp. 21-31, 1993.

[28] J. S. Bendat and A. G. Piersol, Random Data, Analysis and Measurement Procedures, 4th ed. New York: John Wiley \& Sons, 2010.

[29] R. Brincker, L. Zhang, and P. Andersen, "Modal Identification from Ambient Responses Using Frequency Domain Decomposition," International Modal Analysis Conference (IMAC 18), February 2000, pp. 625-630.

[30] R. Brincker, C. E. Ventura, and P. Andersen, "Damping Estimation by Frequency Domain Decomposition," International Modal Analysis Conference (IMAC 19), February 2001, pp. 698-703.

[31] R. Brincker and P. Andersen, Method for Vibration Analysis, U.S. Patent US 6,779,404 B1, August $24,2004$.

[32] Y. Zhang, Z. Zhang, X. Xu, and H. Hua, “Modal Parameter Identification Using Response Data Only,” Journal of Sound and Vibration, vol. 282, no. 1-2, pp. 367-380, April 2005.

[33] L. Zhang, T. Wang, and Y.Tamura "A Frequency-Spatial Domain Decomposition (FSDD) Technique for Operational Modal Analysis," Mechanical Systems and Signal Processing, vol. 24, no. 5, pp. 1227-1239, July 2010.

[34] T. Wang, O. Celik, F. N. Catbas, and L. M. Zhang, "A Frequency and Spatial Domain Decomposition Method for Operational Strain Modal Analysis and Its Application,” Engineering Structures, vol. 114, pp. 104-112, May 2016.

[35] P. Andersen, R. Brincker, B. Peeters, G. De Roeck, L. Hermans, and C. Krämer, "Comparison of System Identification Methods Using Ambient Bridge Test Data,” International Modal Analysis Conference (IMAC 17), February 1999, pp. 1035-1041.

[36] M. Batel, “Operational Modal Analysis - Another Way of Doing Modal Testing,” Sound and Vibration, vol. 36, no. 8, pp. 22-27, August 2002.

[37] C. Devriendt and P. Guillaume, "Identification of Modal Parameters from Transmissibility Measurements," Journal of Sound and Vibration, vol. 314, no. 1-2, pp. 343-356, July 2008.

[38] S. Gade, N. B. Møller, H. Herlufsen, and H. Konstantin-Hansen, "Frequency Domain Techniques for Operational Modal Analysis," 1st International Operational Modal Analysis Conference (IOMAC 2005), May 2005, pp. 261-271.

[39] F. Magalhães, R. Brincker, and Á. Cunha, "Damping Estimation using Free Decays and Ambient Vibration Tests," 2nd International Operational Modal Analysis Conference (IOMAC 2007), May 2007, pp. 513-521.

[40] S. S. Rao, Mechanical Vibrations, 5th ed. New York: Prentice-Hall, 2011.

[41] P. Verboven, "Frequency-Domain System Identification for Modal Analysis," Ph.D. dissertation, Department of Mechanical Engineering, Vrije Universiteit Brussel, Brussels, Belgium, 2002.

[42] P. Guillaume, P. Verboven, and S. Vanlanduit, "Frequency-Domain Maximum Likelihood Identification of Modal Parameters with Confidence Intervals,” International Seminar on Modal Analysis, September 1998, pp. 359-366.

Copyright $($ by the authors. Licensee TAETI, Taiwan. This article is an open access article distributed under the terms and conditions of the Creative Commons Attribution (CC BY-NC) license (https://creativecommons.org/licenses/by-nc/4.0/). 